\title{
Permanence of the periodic predator-prey-mutualist system
}

\author{
Liya Yang ${ }^{1 *}$, Xiangdong Xie ${ }^{2}$, Fengde Chen ${ }^{2}$ and Yalong Xue
}

\section{"Correspondence:}

1378440401@qq.com

'College of Mathematics and

Computer Science, Fuzhou

University, Fuzhou, Fujian 350116,

P.R. China

Full list of author information is

available at the end of the article

\begin{abstract}
In this paper, we study the permanence and the periodic solution of the periodic predator-prey-mutualist system. It is well known that mutualist species can reduce the capture rate of the predator species to the prey species. By further developing the analysis technique of Teng, a set of conditions which ensure the permanence of the system are obtained. In addition, sufficient conditions are derived for the existence of positive periodic solutions to the system. An example together with its numerical simulation shows the feasibility of the main results.
\end{abstract}

MSC: 34C25; 92D25; 34D20; 34D40

Keywords: predator-prey-mutualist system; permanence; periodic solution

\section{Introduction}

As was pointed out by Berryman [1], the dynamic relationship between predator and prey has long been and will continue to be one of the dominant themes in both ecology and mathematical ecology due to its universal existence and importance. Already the predatorprey model has been studied by several scholars [2-10]. For example, Das et al. [8] investigated a three species ecosystem consisting of a prey, a predator, and a top predator. They derived the criteria for local and global stability of all the eight equilibrium points by using a Routh-Hurwitz and Lyapunov function. Wu and Li [9] studied the permanence and global attractivity of the discrete predator-prey system with Hassell-Varley-Holling type III functional response. Chen and Chen [10] proposed a ratio-dependent predator-prey model incorporating a prey refuge. They studied the global stability, limit cycle, and Hopf bifurcation of the system.

Mutualism is one of the most important relationships in the real world, for instance, ants prevent herbivores from feeding on plants (see [11]) and ants prevent predators from feeding on aphids (see [12, 13]). As was pointed out by Murray [14]: 'this area has not been as widely studied as the others even though its importance is comparable to that of predator-prey and competition interactions.' To this end, Rai and Krawcewicz [15] proposed the following three species predator-prey-mutualist system:

$$
\begin{aligned}
& \frac{d x}{d t}=\alpha x\left(1-\frac{x}{K}\right)-\frac{\beta x z}{1+m y}, \\
& \frac{d y}{d t}=\gamma y\left(1-\frac{y}{l x+L_{0}}\right),
\end{aligned}
$$

(c) 2015 Yang et al. This article is distributed under the terms of the Creative Commons Attribution 4.0 International License (http://creativecommons.org/licenses/by/4.0/), which permits unrestricted use, distribution, and reproduction in any medium, provided you give appropriate credit to the original author(s) and the source, provide a link to the Creative Commons license, and indicate if changes were made. 


$$
\frac{d z}{d t}=z\left(-s+\frac{c \beta x}{1+m y}\right)
$$

where $x(t), y(t), z(t)$ denote the densities of prey, mutualist and predator population at any time $t$, respectively. They applied the equivariant degree method to study the Hopf bifurcation phenomenon of the system.

In this paper, we will study the non-autonomous case of system (1.1), i.e.,

$$
\begin{aligned}
& \dot{x}=x\left(a_{1}(t)-b_{1}(t) x-\frac{c_{1}(t) z}{d_{1}(t)+d_{2}(t) y}\right), \\
& \dot{y}=y\left(a_{2}(t)-\frac{y}{d_{3}(t)+d_{4}(t) x}\right), \\
& \dot{z}=z\left(-a_{3}(t)+\frac{c_{2}(t) x}{d_{1}(t)+d_{2}(t) y}\right),
\end{aligned}
$$

where $x$ is the density of the prey at time $t, y$ is the density of the mutualist and $z$ is the density of the predator at time $t$, respectively. $a_{i}(t)(i=1,2,3), d_{j}(t)(j=1,2,3,4)$, and $c_{k}(t)$ $(k=1,2)$ are all continuously positive $w$-periodic functions. The assumption of periodicity of the parameters is a way to incorporate the periodicity of the environment (e.g. seasonal effects of weather condition, food supplies, temperature, mating habits, harvesting, etc.). For this system, due to the lack of density restriction of the predator species, one could not investigate the stability property of the system by constructing the suitable Lyapunov function. Hence, to investigate the persistent property of the system has become one of the most important topics, and we try to push forward this topic.

We arrange the rest of the paper as follows: In Section 2, we introduce one lemma and state the main results of this paper. The results are proved in Section 3. In Section 4, a suitable example together with its numeric simulation is present to show the feasibility of the main results. We end this paper by a briefly conclusion. For more works on the nonautonomous predator-prey system, one could refer to [16-19] and the references cited therein.

\section{Statement of the main results}

Let us first consider the logistic equation,

$$
\frac{d u(t)}{d t}=u(t)(\alpha(t)-\beta(t) u(t))
$$

where $\alpha(t)$ and $\beta(t)$ are periodic continuous functions on $R$ with common periodic $w>0$.

Lemma 2.1 [20] If $\beta(t) \geq 0$ for all $t \in R$ and $\int_{0}^{w} \beta(t) d t>0$, then (2.1) has a unique nonnegative w-periodic solution $u^{*}(t)$ which is globally asymptotically stable, that is, $u(t)-u^{*}(t) \rightarrow$ 0 as $t \rightarrow \infty$ for any positive solution $u(t)$ of (2.1). Moreover, if $\int_{0}^{w} \alpha(t) d t>0$, then $u^{*}(t)>0$ for all $t \in R$ and if $\int_{0}^{w} \alpha(t) d t \leq 0$ then $u^{*}(t) \equiv 0$.

Definition 2.2 System (1.2) is said to be permanent if there exist positive constants $\eta_{i}$, $M_{i}, i=1,2,3$, such that

$$
\eta_{1} \leq \liminf _{t \rightarrow \infty} x(t) \leq \limsup _{t \rightarrow \infty} x(t) \leq M_{1},
$$




$$
\begin{aligned}
& \eta_{2} \leq \liminf _{t \rightarrow \infty} y(t) \leq \limsup _{t \rightarrow \infty} y(t) \leq M_{2}, \\
& \eta_{3} \leq \liminf _{t \rightarrow \infty} z(t) \leq \limsup _{t \rightarrow \infty} z(t) \leq M_{3}
\end{aligned}
$$

for any positive solution $(x(t), y(t), z(t))$ of system (1.2).

We first consider the following system:

$$
\dot{u_{1}}=u_{1}\left(a_{1}(t)-b_{1}(t) u_{1}\right) \text {, }
$$

from Lemma 2.1, (2.2) has a unique nonnegative $w$-periodic solution $u_{10}(t)$ which is globally asymptotically stable.

Second, we consider the following system:

$$
\dot{u_{2}}=u_{2}\left(a_{2}(t)-\frac{u_{2}}{d_{3}(t)+d_{4}(t) M^{*}}\right) \text {, }
$$

where $M^{*}=\max _{0 \leq t \leq w}\left\{u_{10}(t)+1\right\}$, from Lemma 2.1, (2.3) also has a unique nonnegative $w$-periodic solution $u_{20}(t)$ which is globally asymptotically stable.

As concerns the persistent property of the system (1.2), we have the following result.

Theorem 2.3 System (1.2) is permanent if

$$
\int_{0}^{w}\left(-a_{3}(t)+\frac{c_{2}(t) u_{10}(t)}{d_{1}(t)+d_{2}(t) u_{20}(t)}\right) d t>0
$$

holds, where $u_{10}(t)$ and $u_{20}(t)$ are the unique positive periodic solution of systems (2.2) and (2.3), respectively.

As a direct corollary of Theorem 2 in [21], from Theorem 2.3, we have the following.

Corollary 2.4 Under the assumption that (2.4) holds, system (1.2) admits of at least one positive w-periodic solution.

\section{Proof of the main result}

We need the following propositions to prove Theorem 2.3.

Proposition 3.1 There exist positive constants $M_{1}$ and $M_{2}$, such that

$$
\limsup _{t \rightarrow \infty} x(t) \leq M_{1}, \quad \limsup _{t \rightarrow \infty} y(t) \leq M_{2}
$$

for all solutions of system (1.2).

Proof Obviously, $R_{+}^{3}=\{(x, y, z) \mid x \geq 0, y \geq 0, z \geq 0\}$ is a positively invariant set of system (1.2). Given any solution $(x(t), y(t), z(t))$ of system (1.2), we have

$$
\dot{x} \leq x\left(a_{1}(t)-b_{1}(t) x\right) .
$$

By Lemma 2.1, the auxiliary equation

$$
\dot{u_{1}}=u_{1}\left(a_{1}(t)-b_{1}(t) u_{1}\right)
$$


has a unique globally attractive positive $w$-periodic solution $u_{10}(t)$. Let $u_{1}(t)$ is the solution of (3.3) with $u_{1}(0)=x(0)$, by the comparison theorem, we have

$$
x(t) \leq u_{1}(t), \quad t \geq 0 .
$$

Moreover, from the global attractivity of $u_{10}(t)$, for every given $\varepsilon(0<\varepsilon<1)$, there exists a $T_{1}>0$, such that

$$
\left|u_{1}(t)-u_{10}(t)\right|<\varepsilon, \quad t \geq T_{1} .
$$

Equation (3.4) combined with (3.5) leads to

$$
x(t)<u_{10}(t)+\varepsilon, \quad t>T_{1} .
$$

Let $M_{1}=\max _{0 \leq t \leq w}\left\{u_{10}(t)+\varepsilon\right\}$, we have

$$
\limsup _{t \rightarrow \infty} x(t) \leq M_{1}
$$

Since $M^{*}=\max _{0 \leq t \leq w}\left\{u_{10}(t)+1\right\}$, there exists a large enough $T_{2} \geq T_{1}$ such that for all $t>T_{2}$, one has

$$
x(t)<M^{*} .
$$

From (3.8), we have

$$
\dot{y} \leq y\left(a_{2}(t)-\frac{y}{d_{3}(t)+d_{4}(t) M^{*}}\right), \quad t \geq T_{2} .
$$

By Lemma 2.1, the auxiliary equation

$$
\dot{u_{2}}=u_{2}\left(a_{2}(t)-\frac{u_{2}}{d_{3}(t)+d_{4}(t) M^{*}}\right)
$$

has a unique globally attractive positive $w$-periodic solution $u_{20}(t)$. Similarly, we find that there is a constant $T_{3}>T_{2}$ such that

$$
y(t)<u_{20}(t)+\varepsilon, \quad t>T_{3} .
$$

Let $M_{2}=\max _{0 \leq t \leq w}\left\{u_{20}(t)+\varepsilon\right\}$, we have

$$
\limsup _{t \rightarrow \infty} y(t) \leq M_{2}
$$

This completes the proof of Proposition 3.1.

Proposition 3.2 There is a universal constant $\alpha>0$ such that

$$
\limsup _{t \rightarrow \infty} z(t)>\alpha .
$$


Proof If (2.4) holds, we can choose the constant $\varepsilon_{1}>0$ such that

$$
\int_{0}^{w}\left(-a_{3}(t)+\frac{c_{2}(t)\left(u_{10}(t)-\varepsilon_{1}\right)}{d_{1}(t)+d_{2}(t)\left(u_{20}(t)+\varepsilon_{1}\right)}\right) d t>0 .
$$

For any constant $\alpha>0$, we consider the following equation:

$$
\frac{d v_{1}}{d t}=v_{1}(t)\left(a_{1}(t)-\frac{c_{1}(t) 2 \alpha}{d_{1}(t)}-b_{1}(t) v_{1}(t)\right)
$$

Owing to $\int_{0}^{w} a_{1}(t) d t>0, \int_{0}^{w}\left(a_{1}(t)-\frac{c_{1}(t) 2 \alpha}{d_{1}(t)}\right) d t>0$ for small enough $\alpha>0$. By Lemma 2.1, (3.15) has a unique positive $w$-periodic solution $v_{1 \alpha}^{*}(t)$ which is globally asymptotically stable. Let $\bar{v}_{1 \alpha}(t)$ be the solution of (3.15) with initial condition $\bar{v}_{1 \alpha}(0)=u_{10}(0)$, where $u_{10}(t)$ is the unique positive periodic solution of (2.2). Hence, for above $\varepsilon_{1}$, there exists a sufficiently large $T_{4}>T_{3}$ such that

$$
\left|\bar{v}_{1 \alpha}(t)-v_{1 \alpha}^{*}(t)\right|<\frac{\varepsilon_{1}}{4} \quad \text { for } t \geq T_{4} .
$$

By the continuity of the solution in the parameter, we have $\bar{v}_{1 \alpha}(t) \rightarrow u_{10}(t)$ uniformly in $\left[T_{4}, T_{4}+w\right]$ as $\alpha \rightarrow 0$. Hence, for $\varepsilon_{1}>0$, there exists a $\alpha_{0}=\alpha_{0}\left(\varepsilon_{1}\right)>0$ such that

$$
\left|\bar{v}_{1 \alpha}(t)-u_{10}(t)\right|<\frac{\varepsilon_{1}}{4} \quad \text { for } t \in\left[T_{4}, T_{4}+w\right], 0<\alpha<\alpha_{0}
$$

So, we have

$$
\left|v_{1 \alpha}^{*}(t)-u_{10}(t)\right|<\frac{\varepsilon_{1}}{2} \quad \text { for } t \in\left[T_{4}, T_{4}+w\right] .
$$

Note that $v_{1 \alpha}^{*}(t)$ and $u_{10}(t)$ are all $w$-periodic, hence

$$
\left|v_{1 \alpha}^{*}(t)-u_{10}(t)\right|<\frac{\varepsilon_{1}}{2} \quad \text { for } t \geq 0,0<\alpha<\alpha_{0} .
$$

Choosing a constant $\alpha_{1}\left(0<\alpha_{1}<\alpha_{0}, 2 \alpha_{1}<\varepsilon_{1}\right)$, we have

$$
v_{1 \alpha_{1}}^{*}(t) \geq u_{10}(t)-\frac{\varepsilon_{1}}{2}, \quad t \geq 0
$$

If $\lim \sup _{t \rightarrow \infty} z(t) \leq \alpha_{1}$, then there exists $\phi \in R_{+}^{3}$ such that

$$
\limsup _{t \rightarrow \infty} z(t, \phi)<\alpha_{1}
$$

where $(x(t, \phi), y(t, \phi), z(t, \phi))$ is the solution of system (1.2) with $\phi(0)=\left(\phi_{1}(0), \phi_{2}(0)\right.$, $\left.\phi_{3}(0)\right)>0$. So, there exists $T_{5}>T_{4}$ such that

$$
z(t)<2 \alpha_{1}<\varepsilon_{1}, \quad t \geq T_{5}
$$

We have

$$
\dot{x} \geq x\left(a_{1}(t)-\frac{c_{1}(t) 2 \alpha_{1}}{d_{1}(t)}-b_{1}(t) x\right) \quad \text { for } t \geq T_{5} .
$$


Let $v_{1}(t)$ be the solution of (3.15) with $\alpha=\alpha_{1}$ and $v_{1}\left(T_{5}\right)=x\left(T_{5}\right)$, then

$$
x(t) \geq v_{1}(t), \quad t \geq T_{5} .
$$

By the global asymptotic stability of $v_{1 \alpha_{1}}^{*}(t)$, for the given $\varepsilon=\frac{\varepsilon_{1}}{2}$, there exists $T_{6} \geq T_{5}$, such that

$$
\left|v_{1}(t)-v_{1 \alpha_{1}}^{*}(t)\right|<\frac{\varepsilon_{1}}{2}, \quad t \geq T_{6} .
$$

So,

$$
x(t) \geq v_{1}(t)>v_{1 \alpha_{1}}^{*}(t)-\frac{\varepsilon_{1}}{2}, \quad t \geq T_{6}
$$

and hence, by using (3.16), it follows that

$$
x(t)>u_{10}(t)-\varepsilon_{1}, \quad t \geq T_{6} .
$$

From (3.8), there exists $T_{7} \geq T_{6}$ such that $y(t)<u_{20}(t)+\varepsilon_{1}$. Therefore, by using (3.17) and (3.19), for $t \geq T_{7}$ it follows that

$$
\dot{z} \geq z(t)\left(-a_{3}(t)+\frac{c_{2}(t)\left(u_{10}(t)-\varepsilon_{1}\right)}{d_{1}(t)+d_{2}(t)\left(u_{20}(t)+\varepsilon_{1}\right)}\right) .
$$

Integrating the above inequality from $T_{7}$ to $t$ yields

$$
z(t) \geq z\left(T_{7}\right) \exp \int_{T_{7}}^{t}\left(-a_{3}(s)+\frac{c_{2}(s)\left(u_{10}(s)-\varepsilon_{1}\right)}{d_{1}(s)+d_{2}(s)\left(u_{20}(s)+\varepsilon_{1}\right)}\right) d s
$$

Thus, from (3.14) it follows that $z(t) \rightarrow \infty$ as $t \rightarrow \infty$. This is a contradiction. This completes the proof of Proposition 3.2.

Proposition 3.3 There is a universal constant $\eta_{2}>0$ such that

$$
\liminf _{t \rightarrow \infty} y(t) \geq \eta_{2}
$$

Proof From the second equation of system (1.2) it follows that

$$
\dot{y} \geq y\left(a_{2}(t)-\frac{y}{d_{3}(t)}\right)
$$

and we consider the following equation:

$$
\dot{u}_{3}=u_{3}\left(a_{2}(t)-\frac{u_{3}}{d_{3}(t)}\right) .
$$

By Lemma 2.1, (3.22) has a unique positive $w$-periodic solution $u_{30}(t)$. Similar to the analysis of (3.4)-(3.7), for $\varepsilon$ enough small, without loss of generality, $\varepsilon<\min _{0 \leq t \leq w} u_{30}(t)$, we find that there is a constant $T_{8}>T_{7}$ such that

$$
y(t) \geq u_{30}(t)-\varepsilon>0, \quad t>T_{8} .
$$


Letting $\eta_{2}=\min _{0 \leq t \leq w}\left\{u_{30}(t)-\varepsilon\right\}$, we have

$$
\liminf _{t \rightarrow \infty} y(t) \geq \eta_{2}
$$

This completes the proof of Proposition 3.3.

Proposition 3.4 There is a universal constant $\eta_{3}>0$ such that

$$
\liminf _{t \rightarrow \infty} z(t) \geq \eta_{3}
$$

Proof Suppose that (3.25) is not true, then there is a sequence $\left\{\phi_{m}\right\} \in R_{+}^{3}$, such that

$$
\liminf _{t \rightarrow \infty} z\left(t, \phi_{m}\right)<\frac{\alpha}{m+1}, \quad m=1,2, \ldots
$$

On the other hand, by Proposition 3.2, we have

$$
\limsup _{t \rightarrow \infty} z\left(t, \phi_{m}\right)>\alpha, \quad m=1,2, \ldots
$$

Hence, there are time sequences $\left\{s_{q}^{m}\right\}$ and $\left\{t_{q}^{m}\right\}$ satisfying

$$
\begin{aligned}
& 0<s_{1}^{m}<t_{1}^{m}<s_{2}^{m}<t_{2}^{m}<\cdots<s_{q}^{m}<t_{q}^{m}<\cdots, \\
& s_{q}^{m} \rightarrow+\infty, \quad t_{q}^{m} \rightarrow+\infty \quad \text { as } q \rightarrow+\infty
\end{aligned}
$$

and

$$
\begin{aligned}
& z\left(s_{q}^{m}, \phi_{m}\right)=\alpha, \quad z\left(t_{q}^{m}, \phi_{m}\right)=\frac{\alpha}{m+1}, \\
& \frac{\alpha}{m+1}<z\left(t, \phi_{m}\right)<\alpha, \quad t \in\left(s_{q}^{m}, t_{q}^{m}\right) .
\end{aligned}
$$

From the third equation of system (1.2) it follows that

$$
\dot{z} \geq-a_{3}(t) z
$$

Obviously, by integrating (3.28) from $s_{q}^{m}$ to $t_{q}^{m}$,

$$
z\left(t_{q}^{m}, \phi_{m}\right) \geq z\left(s_{q}^{m}, \phi_{m}\right) \exp \int_{s_{q}^{(m)}}^{t_{q}^{(m)}}\left(-a_{3}(t)\right) d t
$$

or

$$
\int_{s_{q}^{(m)}}^{t_{q}^{(m)}}\left(a_{3}(t)\right) d t \geq \ln (m+1)
$$

Thus, from the boundedness of $a_{3}(t)$, we have

$$
t_{q}^{(m)}-s_{q}^{(m)} \rightarrow+\infty \quad \text { as } m \rightarrow+\infty .
$$


By (3.14), there are constants $P>0$ and $\gamma>0$, such that, for any $t \geq P$ and $a \geq 0$,

$$
\int_{a}^{a+t}\left(-a_{3}(s)+\frac{c_{2}(s)\left(u_{10}(s)-\varepsilon_{1}\right)}{d_{1}(s)+d_{2}(s)\left(u_{20}(s)+\varepsilon_{1}\right)}\right) d s>\gamma .
$$

For any $m, q$, and $t \in\left[s_{q}^{(m)}, t_{q}^{(m)}\right]$, we have

$$
\dot{x}\left(t, \phi_{m}\right) \geq x\left(t, \phi_{m}\right)\left(a_{1}(t)-\frac{c_{1}(t) \alpha}{d_{1}(t)}-b_{1}(t) x\left(t, \phi_{m}\right)\right) .
$$

Let $v_{1}(t)$ be the solution of (3.15) with the initial condition $v_{1}\left(s_{q}^{(m)}\right)=\alpha$, then by (3.31), we have $x\left(t, \phi_{m}\right) \geq v_{1}(t)$ for all $t \in\left[s_{q}^{(m)}, t_{q}^{(m)}\right]$. By the periodicity of (3.15), it follows that the periodic solution $v_{1 \alpha}^{*}(t)$ is globally uniformly asymptotically stable. Hence, from (3.16), we find that there is a constant $T_{0}>P$, and $T_{0}$ is independent on any $q$ and $m$, such that

$$
v_{1}(t) \geq u_{10}(t)-\varepsilon_{1} \quad \text { for all } t \geq T_{0}+s_{q}^{(m)} .
$$

By (3.29), there is a $N_{0}>0$ such that $t_{q}^{(m)}>s_{q}^{(m)}+2 T_{0}$ for all $m \geq N_{0}$. Hence,

$$
\begin{aligned}
& x\left(t, \phi_{m}\right) u_{10}(t)-\varepsilon_{1}, \\
& y\left(t, \phi_{m}\right)<u_{20}(t)+\varepsilon_{1} \quad \text { for all } t \in\left[s_{q}^{(m)}+T_{0}, t_{q}^{(m)}\right], m \geq N_{0} .
\end{aligned}
$$

Since

$$
\dot{z}\left(t, \phi_{m}\right) \geq z\left(t, \phi_{m}\right)\left(-a_{3}(t)+\frac{c_{2}(t)\left(u_{10}(t)-\varepsilon_{1}\right)}{d_{1}(t)+d_{2}(t)\left(u_{20}(t)+\varepsilon_{1}\right)}\right)
$$

for all $t \in\left[s_{q}^{(m)}+T_{0}, t_{q}^{(m)}\right]$ and $m \geq N_{0}$, by integrating from $s_{q}^{(m)}+T_{0}$ to $t_{q}^{(m)}$, we obtain

$$
\begin{aligned}
z\left(t_{q}^{(m)}, \phi_{m}\right) \geq & z\left(s_{q}^{(m)}+T_{0}, \phi_{m}\right) \\
& \quad \times \exp \int_{s_{q}^{(m)}+T_{0}}^{t_{q}^{(m)}}\left(-a_{3}(t)+\frac{c_{2}(t)\left(u_{10}(t)-\varepsilon_{1}\right)}{d_{1}(t)+d_{2}(t)\left(u_{20}(t)+\varepsilon_{1}\right)}\right) d t .
\end{aligned}
$$

By (3.30), we have

$$
\begin{aligned}
\frac{\alpha}{m+1} & \geq \frac{\alpha}{m+1} \exp \int_{s_{q}^{(m)}+T_{0}}^{t_{q}^{(m)}}\left(-a_{3}(t)+\frac{c_{2}(t)\left(u_{10}(t)-\varepsilon_{1}\right)}{d_{1}(t)+d_{2}(t)\left(u_{20}(t)+\varepsilon_{1}\right)}\right) d t \\
& >\frac{\alpha}{m+1},
\end{aligned}
$$

which is a contradiction. This completes the proof of Proposition 3.4.

Proposition 3.5 There is a universal constant $M_{3}>0$ such that

$$
\limsup _{t \rightarrow \infty} z(t) \leq M_{3}
$$


Proof Choose the constant $M>0$ and small enough $\varepsilon$ such that

$$
\int_{0}^{w}\left(a_{1}(t)-\frac{c_{1}(t) M}{d_{1}(t)+d_{2}(t)\left(M_{2}+\varepsilon\right)}\right) d t<0
$$

We first prove that

$$
\liminf _{t \rightarrow \infty} z(t) \leq M
$$

If (3.34) is not true, then there is a $T_{9} \geq T_{8}$ such that $z(t)>M$ and $y(t) \leq M_{2}+\varepsilon(\varepsilon$ is small enough) for all $t \geq T_{9}$. Since

$$
\dot{x} \leq x\left(a_{1}(t)-\frac{c_{1}(t) M}{d_{1}(t)+d_{2}(t)\left(M_{2}+\varepsilon\right)}-b_{1}(t) x\right) \quad \text { for all } t \geq T_{9} .
$$

By Lemma 2.1 we easily obtain $x(t) \rightarrow 0$ as $t \rightarrow \infty$. Choose a constant $0<\varepsilon_{2}<\eta_{2}$ such that

$$
\int_{0}^{w}\left(-a_{3}(t)+\frac{c_{2}(t) \varepsilon_{2}}{d_{1}(t)+d_{2}(t)\left(\eta_{2}-\varepsilon_{2}\right)}\right) d t<0
$$

Then there is a $T_{10}>T_{9}>0$ such that $x(t) \leq \varepsilon_{2}$ and $y(t)>\eta_{2}-\varepsilon_{2}$ for all $t \geq T_{10}$, and so

$$
\dot{z} \leq z\left(-a_{3}(t)+\frac{c_{2}(t) \varepsilon_{2}}{d_{1}(t)+d_{2}(t)\left(\eta_{2}-\varepsilon_{2}\right)}\right) .
$$

Integrating the above inequality from $T_{10}$ to $t$ leads to

$$
z(t) \leq z\left(T_{10}\right) \exp \int_{T_{10}}^{t}\left(-a_{3}(s)+\frac{c_{2}(s) \varepsilon_{2}}{d_{1}(s)+d_{2}(s)\left(\eta_{2}-\varepsilon_{2}\right)}\right) d s
$$

Hence, we have $z(t) \rightarrow 0$ as $t \rightarrow \infty$ which is a contradiction. Now, if (3.32) is not true, then there is a sequence $\left\{\phi_{m}\right\} \subset R_{+}^{3}$ such that $\limsup _{t \rightarrow \infty} z\left(t, \phi_{m}\right)>(2 M+1) m$ for all $m=1,2, \ldots$ By (3.34), there are time sequences $\left\{s_{q}^{m}\right\}$ and $\left\{t_{q}^{m}\right\}$ satisfying

$$
\begin{aligned}
& 0<s_{1}^{m}<t_{1}^{m}<s_{2}^{m}<t_{2}^{m}<\cdots<s_{q}^{m}<t_{q}^{m}<\cdots, \\
& s_{q}^{m} \rightarrow+\infty, \quad t_{q}^{m} \rightarrow+\infty \quad \text { as } q \rightarrow+\infty
\end{aligned}
$$

and

$$
\begin{aligned}
& z\left(s_{q}^{m}, \phi_{m}\right)=2 M, \quad z\left(t_{q}^{m}, \phi_{m}\right)=(2 M+1) m, \\
& 2 M<z\left(t, \phi_{m}\right)<(2 M+1) m \text { for all } t \in\left(s_{q}^{m}, t_{q}^{m}\right) .
\end{aligned}
$$

Since there is a $T_{1}^{(m)}>0$ such that $x\left(t, \phi_{m}\right)<M_{1}$ for all $t \geq T_{1}^{(m)}$, we have

$$
\dot{z}\left(t, \phi_{m}\right) \leq z\left(t, \phi_{m}\right)\left(-a_{3}(t)+\frac{c_{2}(t) M_{1}}{d_{1}(t)}\right) \quad \text { for all } t \geq T_{1}^{(m)} .
$$


Obviously, there is a $K_{1}(m)>0$ such that $s_{q}^{m}>T_{1}^{(m)}$ for all $q \geq K_{1}(m)$. Hence, we obtain

$$
z\left(t_{q}^{m}, \phi_{m}\right) \leq z\left(s_{q}^{m}, \phi_{m}\right) \exp \int_{s_{q}^{(m)}}^{t_{q}^{(m)}}\left(-a_{3}(t)+\frac{c_{2}(t) M_{1}}{d_{1}(t)}\right) d t \quad \text { for all } q \geq K_{1}(m)
$$

Consequently,

$$
t_{q}^{(m)}-s_{q}^{(m)} \rightarrow \infty \quad \text { as } m \rightarrow \infty, q \geq K_{1}(m)
$$

By (3.35), there is a $p_{1}>0$ such that for any $t \geq p_{1}$ and $a \geq 0$,

$$
\int_{a}^{a+t}\left(-a_{3}(s)+\frac{c_{2}(s) \varepsilon_{2}}{d_{1}(s)+d_{2}(s)\left(\eta_{2}-\varepsilon_{2}\right)}\right) d s<0
$$

By (3.33), there is a $p_{2}>0$ such that for all $t \geq p_{2}$ and $b \geq 0$,

$$
\int_{b}^{b+t}\left(a_{1}(s)-\frac{c_{1}(s) M}{d_{1}(s)+d_{2}(s)\left(M_{2}+\varepsilon\right)}\right) d s<\ln \frac{\varepsilon_{2}}{2 M}
$$

By (3.37), there is a $L>0$ such that $t_{q}^{(m)}-s_{q}^{(m)}>p_{1}+p_{2}$ for all $m \geq L, q \geq K_{1}(m)$. For any $m \geq L, q \geq K_{1}(m)$ and $t \in\left[s_{q}^{(m)}+p_{1}+p_{2}, t_{q}^{(m)}\right]$, we have

$$
\begin{aligned}
\dot{x}\left(t, \phi_{m}\right) & \leq x\left(t, \phi_{m}\right)\left(a_{1}(t)-\frac{c_{1}(t) 2 M}{d_{1}(t)+d_{2}(t)\left(M_{2}+\varepsilon\right)}\right) \\
& \leq x\left(t, \phi_{m}\right)\left(a_{1}(t)-\frac{c_{1}(t) M}{d_{1}(t)+d_{2}(t)\left(M_{2}+\varepsilon\right)}\right) .
\end{aligned}
$$

Hence,

$$
x\left(t, \phi_{m}\right) \leq x\left(s_{q}^{(m)}, \phi_{m}\right) \exp \int_{s_{q}^{(m)}}^{t}\left(a_{1}(s)-\frac{c_{1}(s) M}{d_{1}(s)+d_{2}(s)\left(M_{2}+\varepsilon\right)}\right) d s<\varepsilon_{2} .
$$

Since

$$
\dot{z}\left(t, \phi_{m}\right) \leq z\left(t, \phi_{m}\right)\left(-a_{3}(t)+\frac{c_{2}(t) \varepsilon_{2}}{d_{1}(t)+d_{2}(t)\left(\eta_{2}-\varepsilon_{2}\right)}\right), \quad t \in\left[s_{q}^{(m)}+p_{1}+p_{2}, t_{q}^{(m)}\right] .
$$

we obtain

$$
\begin{aligned}
z\left(t_{q}^{(m)}, \phi_{m}\right) \leq & z\left(s_{q}^{(m)}+p_{1}+p_{2}, \phi_{m}\right) \\
& \quad \times \exp \int_{s_{q}^{(m)}+p_{1}+p_{2}}^{t_{q}^{(m)}}\left(-a_{3}(t)+\frac{c_{2}(t) \varepsilon_{2}}{d_{1}(t)+d_{2}(t)\left(\eta_{2}-\varepsilon_{2}\right)}\right) d t \\
< & (2 M+1) m,
\end{aligned}
$$

which is contradictory with $z\left(t_{q}^{(m)}, \phi_{m}\right)=(2 M+1) m$. Therefore, there is a constant $M_{3}>0$ such that (3.32) holds. 
Proposition 3.6 There is a universal constant $0<\eta<\eta_{2}$ such that

$$
\limsup _{t \rightarrow \infty} x(t)>\eta
$$

and

$$
\int_{0}^{t}\left(-a_{3}(s)+\frac{c_{2}(s) 2 \eta}{d_{1}(s)+d_{2}(s) \eta_{2}}\right) d s<0
$$

hold, where $\eta_{2}$ is obtained in Proposition 3.3.

Proof If $\lim \sup _{t \rightarrow \infty} x(t) \leq \eta$, then by Proposition 3.3, there is a $T_{11}>T_{10}$ such that $x(t)<2 \eta$ and $y(t) \geq \eta_{2}-\varepsilon$ ( $\varepsilon$ is small enough) for all $t \geq T_{11}$. Since

$$
\dot{z}(t) \leq z(t)\left(-a_{3}(t)+\frac{c_{2}(t) 2 \eta}{d_{1}(t)+d_{2}(t)\left(\eta_{2}-\varepsilon\right)}\right) \quad \text { for all } t \geq T_{11},
$$

and since

$$
\int_{0}^{t}\left(-a_{3}(s)+\frac{c_{2}(s) 2 \eta}{d_{1}(s)+d_{2}(s) \eta_{2}}\right) d s<0,
$$

and for a small enough $\varepsilon$,

$$
\int_{0}^{t}\left(-a_{3}(s)+\frac{c_{2}(s) 2 \eta}{d_{1}(s)+d_{2}(s)\left(\eta_{2}-\varepsilon\right)}\right) d s<0,
$$

by integrating from $T_{11}$ to $t$, we can obtain

$$
z(t) \leq z\left(T_{11}\right) \exp \int_{T_{11}}^{t}\left(-a_{3}(s)+\frac{c_{2}(s) 2 \eta}{d_{1}(s)+d_{2}(s)\left(\eta_{2}-\varepsilon\right)}\right) d s .
$$

Hence, inequality (3.38) implies $z(t) \rightarrow 0$ as $t \rightarrow \infty$, which is a contradiction and Proposition 3.6 is proved.

Proposition 3.7 There is a universal constant $\eta_{1}>0$ such that

$$
\liminf _{t \rightarrow \infty} x(t)>\eta_{1}
$$

Proof We first choose the constant $0<\varepsilon_{3}<\eta$ such that

$$
\int_{0}^{w}\left(-a_{3}(t)+\frac{c_{2}(t) \varepsilon_{3}}{d_{1}(t)+d_{2}(t) \eta_{2}}\right) d t<0 .
$$

Here the constants $\eta_{2}>0$ and $\eta>0$ are obtained in Propositions 3.3 and 3.6. Suppose that (3.39) is not true. Then there is a sequence $\left\{\phi_{m}\right\} \subset R_{+}^{3}$ such that $\liminf _{t \rightarrow \infty} x\left(t, \phi_{m}\right)<$ $\frac{\varepsilon_{3}}{m+1}$ for all $m=1,2, \ldots$. By Proposition 3.6, there are two time sequences $\left\{s_{q}^{(m)}\right\}$ and $\left\{t_{q}^{(m)}\right\}$ satisfying the following conditions:

$$
\begin{aligned}
& 0<s_{1}^{m}<t_{1}^{m}<s_{2}^{m}<t_{2}^{m}<\cdots<s_{q}^{m}<t_{q}^{m}<\cdots, \\
& s_{q}^{m} \rightarrow+\infty, \quad t_{q}^{m} \rightarrow+\infty \quad \text { as } q \rightarrow+\infty, m=1,2, \ldots
\end{aligned}
$$


and

$$
\begin{aligned}
& x\left(s_{q}^{m}, \phi_{m}\right)=\varepsilon_{3}, \quad x\left(t_{q}^{m}, \phi_{m}\right)=\frac{\varepsilon_{3}}{m+1}, \\
& \frac{\varepsilon_{3}}{m+1}<x\left(t, \phi_{m}\right)<\varepsilon_{3} \quad \text { for all } t \in\left(s_{q}^{m}, t_{q}^{m}\right) .
\end{aligned}
$$

By Propositions 3.1 and 3.3, there is a $T^{(m)}>0$ such that $z\left(t, \phi_{m}\right) \leq M_{3}$ and $x\left(t, \phi_{m}\right) \leq M_{1}$ for all $t \geq T^{(m)}$, and further there is a $K^{(m)}>0$ such that $s_{q}^{m} \geq T^{(m)}$ for all $q \geq K^{(m)}$. Hence, for any $t \in\left[s_{q}^{m}, t_{q}^{m}\right]$ and $q \geq K^{(m)}$, we have

$$
\dot{x}\left(t, \phi_{m}\right) \geq x\left(t, \phi_{m}\right)\left(a_{1}(t)-b_{1}(t) M_{1}-\frac{c_{1}(t) M_{3}}{d_{1}(t)}\right) .
$$

By integrating from $s_{q}^{m}$ to $t_{q}^{m}$, we obtain

$$
x\left(t_{q}^{m}, \phi_{m}\right) \geq x\left(s_{q}^{m}, \phi_{m}\right) \exp \int_{s_{q}^{(m)}}^{t_{q}^{(m)}}\left(a_{1}(t)-b_{1}(t) M_{1}-\frac{c_{1}(t) M_{3}}{d_{1}(t)}\right) d t .
$$

Consequently,

$$
\ln (m+1) \leq \int_{s_{q}^{(m)}}^{t_{q}^{(m)}}\left(-a_{1}(t)+b_{1}(t) M_{1}+\frac{c_{1}(t) M_{3}}{d_{1}(t)}\right) d t \quad \text { for all } q \geq K^{(m)}
$$

Hence, we obtain

$$
t_{q}^{(m)}-s_{q}^{(m)} \rightarrow \infty \quad \text { as } m \rightarrow \infty, q \geq K^{(m)} .
$$

By (3.40), there is a constant $T_{12}>0$, and $T_{12}$ is independent on any $m$ and $q$, such that

$$
M_{3} \exp \int_{s_{q}^{(m)}}^{t_{q}^{(m)}}\left(-a_{3}(t)+\frac{c_{2}(t) \varepsilon_{3}}{d_{1}(t)+d_{2}(t)\left(\eta_{2}-\varepsilon\right)}\right) d t<\eta_{3}
$$

By (3.41), there is a $M_{0}>0$ such that $t_{q}^{(m)}>s_{q}^{(m)}+M_{0}$ for all $m \geq M_{0}$ and $q \geq K^{(m)}$. Hence, for any $t \in\left[s_{q}^{m}, t_{q}^{m}\right], m \geq M_{0}$, and $q \geq K^{(m)}$, we have $y(t) \geq \eta_{2}-\varepsilon$ and

$$
\dot{z}\left(t, \phi_{m}\right) \leq z\left(t, \phi_{m}\right)\left(-a_{3}(t)+\frac{c_{2}(t) \varepsilon_{3}}{d_{1}(t)+d_{2}(t)\left(\eta_{2}-\varepsilon\right)}\right) .
$$

By integrating from $s_{q}^{(m)}$ to $s_{q}^{(m)}+M_{0}$, we obtain

$$
\begin{aligned}
\eta_{3} & <z\left(s_{q}^{(m)}+M_{0}, \phi_{m}\right) \\
& \leq z\left(s_{q}^{(m)}, \phi_{m}\right) \exp \int_{s_{q}^{(m)}}^{s_{q}^{(m)}+M_{0}}\left(-a_{3}(t)+\frac{c_{2}(t) \varepsilon_{3}}{d_{1}(t)+d_{2}(t)\left(\eta_{2}-\varepsilon\right)}\right) d t<\eta_{3},
\end{aligned}
$$

which is a contradiction, and Proposition 3.7 is proved. 


\subsection{Proof of Theorem 2.3}

The results of Theorem 2.3 now follow from Propositions 3.1-3.7.

\section{Example}

In this section, we shall give an example to illustrate the feasibility of the main results.

Example Consider the following predator-prey-mutualist system (see Figure 1):

$$
\begin{aligned}
& \dot{x}=x\left(3.2-0.3 x-\frac{0.6 z}{0.8+0.3 y}\right), \\
& \dot{y}=y\left(3.0-\frac{y}{0.5+0.5 x}\right), \\
& \dot{z}=z\left(-(0.7-0.1 \sin t)+\frac{0.5 x}{0.8+0.3 y}\right) .
\end{aligned}
$$

Corresponding to system (1.2), one has

$$
\begin{aligned}
& a_{1}(t)=3.2, \quad b_{1}(t)=0.3, \quad c_{1}(t)=0.6, \quad d_{1}(t)=0.8, \\
& d_{2}(t)=0.3, \quad a_{2}(t)=3.0, \quad d_{3}(t)=0.5, \quad d_{4}(t)=0.5, \\
& a_{3}(t)=0.7-0.1 \sin t, \quad c_{2}(t)=0.5 .
\end{aligned}
$$

One easily sees that

$$
\dot{u}_{1}(t)=u_{1}(t)\left(3.2-0.3 u_{1}(t)\right)
$$

has a unique positive periodic solution $u_{10}(t) \approx 10.667$. So $M^{*}=11.667$,

$$
\dot{u}_{2}(t)=u_{2}(t)\left(3.0-\frac{u_{2}(t)}{0.5+0.5 \times 11.667}\right)
$$

has a unique positive periodic solution $u_{20}(t) \approx 17.503$.

By simple computation, one has

$$
\begin{aligned}
& \int_{0}^{w}\left(-a_{3}(t)+\frac{c_{2}(t) u_{10}(t)}{d_{1}(t)+d_{2}(t) u_{20}(t)}\right) d t \\
& \quad=\int_{0}^{2 \pi}\left(-(0.7-0.1 \sin t)+\frac{0.5 \times 10.667}{0.8+0.3 \times 17.503}\right) d t \approx 1.1394>0 .
\end{aligned}
$$

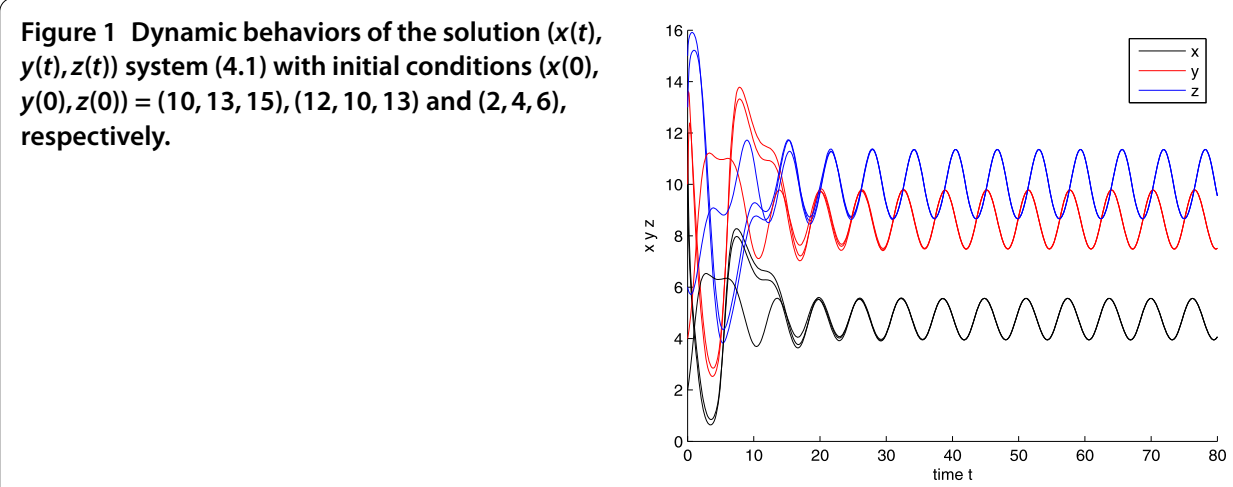


Condition (2.4) is satisfied. Thus, corresponding to Theorem 2.3 and Corollary 2.4, we know that system (4.1) is permanent and admits at least one positive $2 \pi$-periodic solution.

\section{Conclusion}

In this paper, we studied a periodic predator-prey-mutualist system. From system (1.2), we see the mutualist species $y$ can reduce the capture rate of the predator species $z$ to the prey species $x$. By further developing the analysis technique of Teng [20], we obtain a set of conditions which ensure the permanence of system (1.2). Note that $u_{10}(t)$ and $u_{20}(t)$ are the globally attractive periodic solution of (2.2) and (2.3), respectively, which, as shown by Lemma 2.1, always exists. Hence, the left side of condition (2.4) implies that if the death rate of the predator species is enough small and the cooperation effect between species $x$ and $y$ is not very strong, the system is permanent.

\section{Competing interests}

The authors declare that there are no competing interests.

Authors' contributions

The authors read and approved the final manuscript.

\section{Author details}

${ }^{1}$ College of Mathematics and Computer Science, Fuzhou University, Fuzhou, Fujian 350116, P.R. China. ${ }^{2}$ Department of Mathematics, Ningde Normal University, Ningde, Fujian 352300, P.R. China.

\section{Acknowledgements}

The authors are grateful to the anonymous referees for their excellent suggestions, which greatly improved the presentation of the paper. Also, the research was supported by the Natural Science Foundation of Fujian Province (2015J010121, 2015J01019).

Received: 11 June 2015 Accepted: 30 September 2015 Published online: 23 October 2015

\section{References}

1. Berryman, AA: The origins and evolution of predator-prey theory. Ecology 73(5), 1530-1535 (1992)

2. Chen, LJ, Chen, FD, Chen, LJ: Qualitative analysis of a predator-prey model with Holling type II functional response incorporating a constant prey refuge. Nonlinear Anal., Real World Appl. 11(1), 246-252 (2010)

3. Chen, FD, Wu, CQ, Chen, WL: Dynamic behaviors of a Lotka-Volterra predator-prey model incorporating a prey refuge and predator mutual interference. Appl. Math. Comput. 219(15), 7945-7953 (2013)

4. Chen, LJ, Chen, FD, Wang, YQ: Influence of predator mutual interference and prey refuge on Lotka-Volterra predator-prey dynamics. Commun. Nonlinear Sci. Numer. Simul. 18(11), 3174-3180 (2013)

5. $\mathrm{Yu}, \mathrm{SB}, \mathrm{Wu}, \mathrm{HH}$, Chen, JB: Multiple periodic solutions of delayed predator-prey systems with type IV functional responses on time scales. Discrete Dyn. Nat. Soc. 2012, Article ID 271672 (2012)

6. Chen, FD, Zhang, HY: Global asymptotical stability of the positive equilibrium of Lotka-Volterra prey-predator model incorporating a constant number of prey refuge. Nonlinear Anal., Real World Appl. 13(6), 2790-2793 (2012)

7. Chen, LJ, Xu, JY, Li, Z: Permanence and global attractivity of a delayed discrete predator-prey system with general Holling-type functional response and feedback controls. Discrete Dyn. Nat. Soc. 2008, Article ID 629620 (2008)

8. Das, K, Shiva Reddy, K, Srinivas, MN, Gazi, NH: Chaotic dynamics of a three species prey-predator competition model with noise in ecology. Appl. Math. Comput. 231, 117-133 (2014)

9. Wu, RX, Li, L: Permanence and global attracticity of the discrete predator-prey system with Hassell-Varley-Holling III type functional response. Discrete Dyn. Nat. Soc. 2013, Article ID 393729 (2013)

10. Chen, LJ, Chen, FD: Global stability and bifurcation of a ratio-dependent predator-prey model with prey refuge. Acta Math. Sin. Chin. Ser. 57(2), 301-310 (2014) (in Chinese)

11. Bently, BL: Extrafloral nectarines and protection by pugnacious bodyguards. Annu. Rev. Ecol. Syst. 8, 407-427 (1977)

12. Addicott, JF: A multispecies aphid-ant association: density dependence and species-specific effects. Can. J. Zool. 57(3), 558-569 (1979)

13. Way, MJ: Mutualism between ants and honeydew-producing homoptera. Annu. Rev. Entomol. 8(1), 307-344 (1963)

14. Murray, JD: Mathematical Biology. Springer, Berlin (1988)

15. Rai, B, Krawcewicz, W: Hopf bifurcation in symmetric configuration of predator-prey-mutualist systems. Nonlinear Anal., Real World Appl. 71(9), 4279-4296 (2009)

16. Yang, $L Y, X i e, X X, W u, C Q:$ Periodic solution of a periodic predator-prey-mutualist system. Commun. Math. Biol. Neurosci. 2015, Article ID 7 (2015)

17. Chen, FD, Shi, CL: Global attractivity in an almost periodic multi-species nonlinear ecological model. Appl. Math. Comput. 180(1), 376-392 (2006)

18. Yu, SB, Chen, FD: Almost periodic solution of a modified Leslie-Gower predator-prey model with Holling-type II schemes and mutual interference. Int. J. Biomath. 3, 81-95 (2014) 
19. Shi, CL, Li, Z, Chen, FD: Extinction in a nonautonomous Lotka-Volterra competitive system with infinite delay and feedback controls. Nonlinear Anal., Real World Appl. 13(5), 2214-2226 (2012)

20. Teng, Z: Uniform persistence of the periodic predator-prey Lotka-Volterra system. Appl. Anal. 72(3), 339-352 (1999)

21. Teng, Z, Chen, L: The positive periodic solutions of periodic Kolmogorov type systems with delays. Acta Math. Appl. Sin. 22, 446-456 (1999) (in Chinese)

Submit your manuscript to a SpringerOpen ${ }^{\circ}$ journal and benefit from:

- Convenient online submission

- Rigorous peer review

- Immediate publication on acceptance

- Open access: articles freely available online

- High visibility within the field

- Retaining the copyright to your article 